\title{
Glaucoma simplex and the management of first-degree relatives
}

\author{
S. J. H. MILLER \\ Moorfields Eye Hospital, High Holborn, London W.C.I
}

During the last Io years a sibling clinic has been held at Moorfields Eye Hospital, High Holborn, and the staff has been encouraged to welcome first-degree relatives (parents, $\frac{0}{O}$. siblings, and children) of patients suffering from established simple glaucoma for further or investigation; this has led us to the problem of when to initiate therapy.

The criteria for the diagnosis of simple glaucoma has been defined as follows by Hollows and Graham (i $966 a, b)$ :

(a) Characteristic visual field defects;

(b) Glaucomatous cupping;

(c) Intraocular pressure above or equal to or known to have been above or equal to $2 \mathrm{I} \mathrm{mm} . \mathrm{Hg}$;

(d) Anterior chamber-angle free of abnormal mesoderm and unobstructed.

There are no difficulties in decision-making in regard to item $(d)$, but the other three need some amplification.

\section{(a) Early field loss in simple glaucoma}

Baring of the blind spot is discounted as an early sign. It occurs in 16 per cent. of normal individuals over the age of 40 years (Armaly, $1967 \mathrm{e}$ ) and can be manipulated by varying the strength of the stimulus employed.

Enlargement of the blind spot is a late sign, as is the development of a nasal step in the peripheral field (Aulhorn and Harms, i 967 ).

Concentric contraction of the visual field is not an early sign and if it is found in a case of suspected glaucoma it is important to eliminate lens opacities as a possible cause.

The field defects which are characteristic of early glaucoma are small scotomata lying $\bigcirc$ in the Bjerrum area, which later enlarge, become confluent, and join with the blind spot.

Such scotomata are often absolute and quite near fixation and are well produced on repeated examinations. The difficulty lies in finding them at all, and static perimetry o with variable target luminance is a superior method to the commonly employed kinetic perimetry.

First-degree relatives should always be examined with variable additions of $0 \cdot 5$ dioptre, both plus and minus (Armaly, I $967 \mathrm{e}$ ), to avoid mistaking a refraction scotoma for a nerve fibre bundle defect.

\section{(b) Cupping}

The difficulty in classifying a disc as glaucomatous is lack of precision in regard to the characteristics of early pathological cupping, and a physiological cup may gradually 
change to a pathological cup so that it may be quite impossible to draw a firm conclusion at a certain stage of development.

More emphasis must be placed on asymmetry of disc appearance (contrasting it with the fellow-disc) and on temporal disc changes (meaning changes with time) than on minutiae of observation at any particular moment.

Asymmetry of the cup/disc ratio is not limited to the later stages of the disease (Armaly, 1970b). In individuals with monocular involvement of the visual field, Armaly found that he could have suspected glaucoma on the basis of inequality in 69 per cent. of the group by careful ophthalmoscopic examination and successfully identified the involved eye in 63 per cent. He found that a difference $>0.2$ in the cup/disc ratio occurs in normal eyes in less than I per cent. of the population. This asymmetry may be seen in cases of unilateral secondary glaucoma and of unilateral chronic simple glaucoma.

Qualitative changes which occur at the disc head with time are assessed under the following headings:

(i) Colour;

(ii) Extent of cupping;

(iii) Depth of cupping; (iv) Displacement of vessels nasally;

(v) Marginal haemorrhages; (vi) Peripapillary halo:

(vii) Fluorescein angiography.

CASE REPORT

In 1966 a male patient aged 43 years presented himself as a first-degree relative with applanation tensions of $34 \mathrm{~mm} . \mathrm{Hg}$. The optic discs were photographed (Fig. I) and the visual fields were assessed and found to be full. He failed to keep his follow-up appointments or accept advice on treatment but, in response to a personal appeal, he returned for further examination 4 years later. The discs were found to have changed by becoming paler and more cupped in width and depth, the vessels had become displaced nasally, and a peripapilary halo had become evident (Fig. 2). The visual fields showed a related deterioration (Fig. 3).

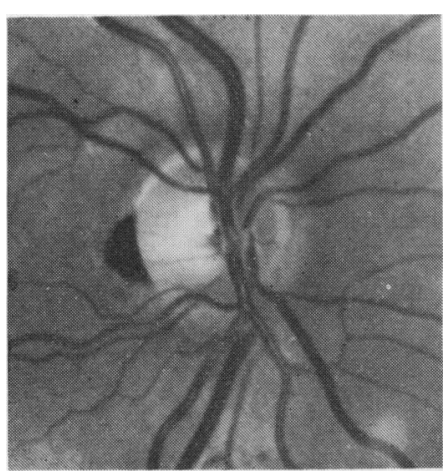

FIG. I Right optic disc photographed in 1966

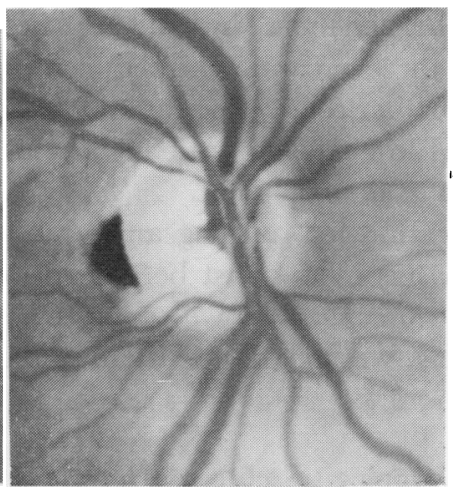

FIG. 2 Right optic disc photographed in $\mathbf{1 9 7 0}$

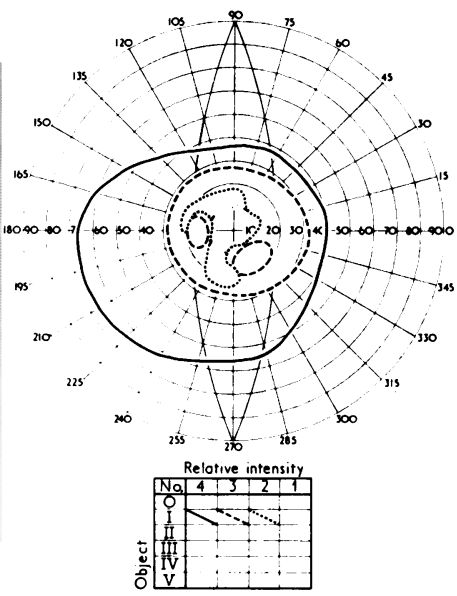

FIG. 3 Right visual field, showing arcuate scotoma in 1970

Hollows and Graham (1966a,b) found that ophthalmoscopy was an efficient detector of established glaucoma in the hands of the ophthalmologist. They noted a total of 102 discs meriting further investigations in their survey and to their satisfaction this group proved to contain all fourteen of the patients who had previously shown unknown glaucoma- 
like field defects. Hollows and McGuinness (1967), in a survey of the cup size in a normal population, came to the conclusion that its distribution was unaffected by sex, age, intra-은

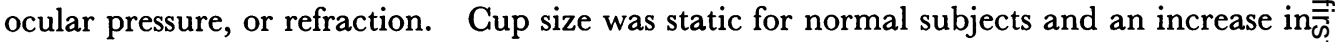
size always indicated a departure from normality.

Fisher, Carpenter, and Wheeler (1970) have shown statistically how closely the simple subjective assessment of cupping of the disc is correlated with the size of the visual field $\frac{\bar{c}}{5}$ in established glaucoma, and Aulhorn and Harms (1960), by a study of the pattern of optic nerve atrophy and disturbances in the capillary circulation at the nerve head, weres able to predict the topography of visual field defects in 53 out of 74 cases.

I should like to take this idea further and to suggest that a change in appearance at the optic nerve head is probably the best predictor of impending field loss or of further deteriora-o tion of the visual field.

\section{(c) Tension}

If a patient is found with a characteristic visual field defect, glaucomatous cupping $\$$ and a tension below $21 \mathrm{~mm} . \mathrm{Hg}$, monitoring of tension is important in order to establish the precise diagnosis and eliminate peaks of raised pressure.

Ideally, monitoring should be done during working hours by asking the patient to report at various times of the day so that a pattern of diurnal variation during an average working day may be gradually acquired. To complete the 24-hour cycle a night in hospital is necessary to take tensions during non-working hours, particularly on rising.

\section{TREATMENT}

What treatment, if any, should be recommended for patients who have a field defect, cupping, and tension below $2 \mathrm{I} \mathrm{mm} . \mathrm{Hg}$ at all times? If pilocarpine reduces the tension significantly-by ro per cent., then treatment is given. The reasoning behind thiso recommendation is that lowering of tissue pressure in the presence of a hypoxic nerve head is likely to be beneficial in assisting the long-term survival of the visual field. If tissue pressure in the eye cannot be reduced by therapy, there is no point in giving it, as miotics will only further reduce the functional field of vision. The important investigation:in the follow-up of low tension patients is pressure-monitoring, and a rise in tension calls for a modification of management.

The main indication for treating an individual who presents himself with evidence of glaucoma simplex has in the past been field loss in the presence of a cupped disc and a tension above $21 \mathrm{~mm} . \mathrm{Hg}$. As we move to a consideration of first-degree relatives who present no field loss, we may divide them into a number of sub-groups.

(I) Full field

(2) Full field Glaucomatous cupping $(>0 \cdot 6) \quad$ Normotensives with cupping Tension below $20 \mathrm{~mm} . \mathrm{Hg}$

(3) Full field

Normal disc $(<0 \cdot 6)$

Tension greater than $20 \mathrm{~mm} . \mathrm{Hg}$ 
(x) Patients with full fields, cupping which is regarded as pathological, and a tension greater than $20 \mathrm{~mm} . \mathrm{Hg}$ must be treated, and in following such patients serial photography of the optic disc is likely to prove the most sensitive method of detecting the possibility of impending field loss.

(2) Patients with full fields, glaucomatous cupping, and tensions below $20 \mathrm{~mm} . \mathrm{Hg}$ should be carefully and periodically screened, a particularly close watch being kept on changes in the optic disc.

(3) Those who show full fields, discs within normal limits (cupping 0.6 or below), and an intraocular pressure of above $2 \mathrm{I} \mathrm{mm.Hg}$ at some time in a 24-hour period are classified as ocular hypertensives, and many of them show no progression when watched over the years (Linnér, 1967). Such patients must, of course, be placed under periodical review but, again, the meaningful indicator of impending field decay is a change at the optic nerve head heralding optic atrophy. It is obvious that both fields and tensions must be assessed, but a change at the disc head in the presence of the same tension and a maintained field calls for treatment and lowering of tissue pressure by glaucoma therapy.

In all three groups a change in the optic nerve head is the all-important sign, not only as a guide to the effectiveness of treatment as in Group I but as an indication to initiate therapy in Groups 2 and 3.

How are we to advise first-degree relatives who show no evidence of simple glaucoma? Linnér ( 1967 ) demonstrated how dangerous it would be to dismiss them with the assurance that they were not prone to this disease. He found, in his series of cases examined with Stromberg, that most of the eyes with moderate ocular hypertension initially, retained the same pressure over the next 5 years with no deterioration in function, whereas another group with normal tension initially developed a rise over the same period leading to frank glaucoma. Again, in this group of apparently healthy first-degree relatives, followup examinations of the optic disc are as important or even more important than tension readings.

\section{Conclusion}

It has been abundantly shown that the ophthalmoscope is the best screener for established simple glaucoma (Hollows and Graham, ig66a,b). The tonometer alone or with tonography has proved a somewhat poor predictor of functional loss. Permanent glaucomatous field changes are always preceded by changes at the optic disc which are recognizable as pathological. If the examination of first-degree relatives is employed as a first screening device, then careful attention to change at the optic disc is likely to prove a better predictor of impending functional field loss than tonometry or tonography and a more compelling indicator for treatment. This can be achieved only by regular periodic photography of the optic disc.

The regular clinical examination of first-degree relatives has led to the diagnosis of glaucoma at a significantly earlier age. Miotic therapy has many disadvantages when accommodation is still active. This has stimulated us to search for a means of controlling tension without producing disabling myopia and miosis. Dr. Paterson will give an account in the following paper of a series of drug trials undertaken with this aim in mind. 